\title{
Avaliação de processo em psicoterapia psicanalítica na adolescência
}

\author{
Silvia Pereira da Cruz Benetti \\ Maria Cristina Vieweger de Mattos \\ Nathália Bohn da Silva, Graduanda \\ Aline Alvares Bittencourt \\ Universidade do Vale do Rio dos Sinos, RS, Brasil
}

\begin{abstract}
Resumo
As taxas de abandono psicoterápico na infância e adolescência são altas, entretanto, são poucos os trabalhos dirigidos para essas faixas etárias. Este artigo investiga o processo terapêutico de um caso de abandono de psicoterapia psicanalítica de uma adolescente. Para tal, foram avaliadas 20 sessões da psicoterapia através do Adolescent Psychotherapy Q-Set (APQ), instrumento que descreve atitudes e comportamentos do paciente e do terapeuta, bem como das interações terapeuta/paciente nas sessões terapêuticas. A análise do APQ foi integrada aos aspectos dinâmicos da paciente e associada às anotações clínicas fornecidas pela terapeuta para a elaboração do entendimento clínico do processo. Concluiu-se que a não aquisição de insight da paciente, aliado a um estilo defensivo imaturo constituíram fatores preditivos ao abandono psicoterápico.
\end{abstract}

Palavras-chave: adolescência; avaliação terapêutica; psicoterapia psicanalítica.

\section{Process evaluation in psychoanalytic psychotherapy in adolescence}

\begin{abstract}
Dropout rates from psychotherapy among children and adolescents are high, however there fewer studies targeting these age groups. This paper investigates the therapeutic process of a dropout case in psychoanalytic psychotherapy in adolescence. For this purpose, 20 sessions of psychotherapy were evaluated using the Adolescent Psychotherapy Q-Set (APQ), an instrument that describes attitudes and behaviors of the patient and the therapist, and the therapist/patient interactions during the therapeutic sessions. The analysis of the instrument was integrated into the dynamic aspects of the patient and also associated with clinical notes provided by the therapist to elaborate the clinical understanding of the process. It was concluded that the impossibility of the patient's insight acquisition combined with an immature defensive style, constituted predictive factors to psychotherapy dropout. Therefore, compared to many possibilities of understanding this phenomenon, there is the importance of conducting researches aimed at in-depth analysis of psychotherapeutic processes.
\end{abstract}

Keywords: adolescence; therapeutic evaluation; psychoanalytic psychotherapy.

\section{Proceso de evaluación en la psicoterapia psicoanalítica en la adolescencia} Resumen

Las tasas de abandono de la psicoterapia Entre los niños y adolescentes son altos, sin embargo, hay pocos estudios dirigidos a estos grupos. El presente artículo investiga el processo terapêutico de um caso de abandono en psicoterapia psicoanalítica en la adolescência. Para tal se evaluaron 20 sesiones de psicoterapia por médio del Adolescent Psychotherapy Q-Set (APQ), instrumento que describe actitudes y conductas del paciente y del terapeuta, asi como las interacciones terapeuta/paciente durante las sesiones terapêuticas. El analisis del APQ fue integrada a los aspectos dinâmicos de la paciente y associada a las anotaciones clínicas realizadas por la terapeuta para la elaboracion del entendimento clínico del proceso. Se concluye que la no aquicision de insight por parte de la paciente, aliado a un estilo defensivo inmaturo constituyeron facteres predictivos al abandono terapêutico. Desta forma, frente a los diversos vértices de comprensión de este fenômeno, se verifica la importância de la realizacion de pesquisas que el analisis en profundidad de los procesos psicoterápicos.

Palabras clave: adolescência; evaluación terapêutica; psicoterapia psicoanalitica. 


\section{Introdução}

A pesquisa em psicoterapia psicanalítica tem ampliado seu espaço no meio científico a partir da publicação de uma série de revisões importantes e meta análises sobre os resultados das intervenções psicoterápicas nessa abordagem teórica. Nesse caso, os estudos confirmam a efetividade e a eficácia das psicoterapias psicanalíticas em distintos quadros clínicos e faixas etárias. Entretanto, o foco das pesquisas foi basicamente com pacientes adultos, apontando que os ganhos obtidos com a psicoterapia psicanalítica se assemelham a de outras abordagens terapêuticas anteriormente pesquisadas (Gerber et al., 2011; Shedler, 2012). No que se refere à pesquisa em psicoterapia psicanalítica na adolescência, um número menor de estudos foram realizados (Edlund, \& Carlberg, 2016). Déficit que pode ser explicado pelo fato de haver um número menor de medidas destinadas a avaliar os processos psicoterápicos nesta faixa etária, o que leva a maioria das investigações a utilizar modelos de adultos, sem levar em consideração as diferenças evolutivas e as especificidades do período (Stewart, Steele, \& Roberts, 2012).

As características da adolescência justificam um olhar atento às questões próprias desta etapa. Conforme a Organização Mundial da Saúde (OMS, 2015), a adolescência é um período evolutivo compreendendo as idades entre 12 a 19 anos. Pesquisas destacam a necessidade de prevenção e intervenção e apontam uma alta prevalência de transtornos emocionais nesse período. A eclosão de quadros psicopatológicos ocorre em função de alguns adolescentes não possuírem condições de responder positivamente às exigências do processo evolutivo (Merikangas et al., 2010; Valverde, Vitalle, Sampaio, \& Schoen, 2012), já que a consolidação da identidade exige internamente do adolescente um afastamento de objetos primários, ligados às figuras parentais (Adesse, Santos, \& Cardoso, 2014).

Considerando o aumento de sofrimento psíquico característico da adolescência e as possibilidades de ressignificação e elaboração de conflitivas psíquicas, esta fase contempla um momento oportuno para intervenções psicoterápicas (Macedo et al., 2011). Nesse sentido, recentes estudos reforçam a eficácia e a efetividade das psicoterapias nesta etapa e apontam melhoras comportamentais nos adolescentes em tratamento (Weitkamp et al., 2014).

Apesar desse progresso das pesquisas em psicoterapia psicanalítica nas distintas faixas etárias, há um número menor de trabalhos dirigidos para infância $\mathrm{e}$ adolescência, quando comparados com investigações baseadas na abordagem cognitivo-comportamental (Palmer, Nascimento, \& Fonagy, 2013). Nessa direção, Midgley (2008), delimita dois aspectos fundamentais de investigação focalizando essas faixas etárias: estudos sobre o porquê a psicoterapia funciona e sobre como ela funciona em diferentes contextos, principalmente destacando a descrição dos processos terapêuticos. Estudos de processo em psicoterapia permitem a compreensão de como e porque as mudanças terapêuticas ocorrem, permitindo maior profundidade no entendimento dos fatores envolvidos nos processos de mudança.

Recentemente, um instrumento (Adolescent Psychotherapy Q-Set, APQ) foi adaptado para a adolescência (Calderón, 2014). Derivado do Psychotherapy Process Q-Set (PQS, Jones, 2000), o APQ é um instrumento baseado na metodologia $\mathrm{Q}$, composto por 100 itens que avaliam aspectos do terapeuta, do paciente e da interação, durante a sessão terapêutica. O método Q constitui-se em um grupo de afirmações sobre um determinado tópico e sobre os quais os sujeitos são solicitados a classificar cada uma das afirmações, variando desde +5 , concordância total com a afirmação, até -5 , discordância total. $\mathrm{O}$ método permite que se correlacione respostas com um protótipo ideal, tal como no caso de relacionar respostas de determinados sujeitos com aquelas de um expert sobre um tema (Bigras \& Dessen, 2002).

Assim, cada item do instrumento descreve um aspecto do processo terapêutico através da avaliação das falas e comportamentos da dupla, possibilitando observar objetivamente a presença, ausência ou relevância destes itens (Calderón, 2014). Além disso, o instrumento permite aliar o estudo empírico do processo com a integração da compreensão dinâmica do caso. Essa possibilidade de explorar o aspecto descritivo da sessão, integrado ao entendimento dinâmico do caso, complementa a compreensão dos processos terapêuticos. Dessa forma, o APQ viabiliza o estudo de fenômenos comuns e específicos nas psicoterapias, pouco estudados na fase da adolescência.

Um desses fenômenos é o abandono de tratamento (Ormhaug, \& Jensen, 2016, Gatta et al., 2010). No caso, o abandono refere-se a decisão unilateral do paciente em parar de comparecer à terapia, com ou sem o conhecimento prévio do terapeuta. Esta tem sido a definição mais amplamente utilizada nos estudos desse fenômeno e foi adotada como referência neste artigo (Gastaud, Basso, Soares, Eizirik, \& Nunes, 2011).

Diversos fatores têm sido associados ao abandono terapêutico, incluindo características do paciente, tais como idade, escolaridade, e sintomatologia, bem como fatores do tratamento, modalidade terapêutica, 
características do terapeuta. As taxas de abandono entre crianças e adolescentes apontadas na literatura internacional indicam a gravidade do fenômeno. $\mathrm{Na}$ meta análise de Haan, Boon, Jong, Hoeve e Vermeiren (2013), foi identificado um percentual de até 75\% de abandono nessas faixas etárias. $\mathrm{Na}$ adolescência o abandono terapêutico assume uma característica peculiar ao período, visto que é potencializado pela própria busca da autonomia do jovem associada à consolidação da identidade.

Frente à necessidade de estudar fenômenos comuns às psicoterapias, este artigo focaliza o estudo de processo de um caso de abandono terapêutico de uma adolescente atendida em psicoterapia psicanalítica. Para tal, propõe-se uma integração de dados empíricos com observações clínicas mais tradicionais, buscando diminuir a lacuna existente entre a prática clínica e a pesquisa (Serralta, Nunes, \& Eizirik, 2011). Embora se reconheça a importância de delineamentos investigativos que privilegiam a randomização amostral e o controle das variáveis intervenientes, pesquisas qualitativas, organizadas em amostras com menor número de participantes, têm uma contribuição importante para o estudo das psicoterapias. Esse ponto é de fundamental interesse nos estudos de processo, que visam ao entendimento do que ocorre no aqui e agora das sessões terapêuticas. Delineamentos dessa ordem, principalmente na modalidade de estudos de casos sistemáticos, podem garantir a investigação de processo em profundidade, ao mesmo tempo em que é feita a análise empírica das evidências das mudanças terapêuticas (McLeod \& Elliott, 2011).

\section{Método}

\section{Delineamento}

Foi utilizado como metodologia o estudo de caso sistemático com um delineamento misto. $\mathrm{O}$ estudo de caso sistemático faz uso de medidas objetivas aplicadas a sessões de psicoterapia filmadas (Serralta, Nunes, \& Eizirik, 2011).

\section{Participantes}

Paciente: Juliana ${ }^{1}, 18$ anos, realizou triagem início de 2013 em uma clínica psiquiátrica, queixandose de irritabilidade e sintomas depressivos que a acompanhavam desde a infância. Referiu já ter recebido diagnóstico de transtorno de déficit de atenção e hiperatividade (TDAH). Os pais de Juliana divorciaram-se quando ela contava 3 anos de idade, passando ela e o irmão mais velho a morar com a mãe

\footnotetext{
1 Nome fictício.
}

que apresentava transtorno de humor bipolar. O pai era alcoolista e atualmente apresenta diagnóstico de depressão.

Seus relatos da infância evidenciavam completa ausência das figuras parentais. A mãe, segundo a própria paciente, nunca soube cuidar, além de passar horas fora de casa. Quando presente, brigava e reclamava muito dos filhos, agredindo-os fisicamente.

Durante o início de sua adolescência, fazia uso abusivo de álcool e maconha. A mãe era ciente destes ocorridos, visto que a jovem eventualmente fazia uso destas substâncias em casa. Aos 13 anos teve seu primeiro envolvimento afetivo com um rapaz 10 anos mais velho. Atualmente, está em outro relacionamento com um rapaz de 23 anos, diagnosticado com transtorno de humor bipolar com sintomas controlados pelo uso de medicação.

Juliana abandonou a escola no ano de 2012, indo trabalhar em uma empresa de entrega. No início de 2013, buscou atendimento em uma clínica privada onde foi encaminhada para psicoterapia individual de orientação psicanalítica e também para avaliação psiquiátrica. O diagnóstico realizado pelo psiquiatra durante a etapa inicial do tratamento psicoterápico, utilizando-se do modelo proposto para diagnóstico multiaxial do DSM-IV-TR (APA, 2002) foi de transtorno depressivo maior.

Terapeuta: Possui treinamento formal em psicoterapia de orientação psicanalítica, cujo modelo é baseado na tríplice formação: seminários teóricos, supervisões e tratamento pessoal. Tem 33 anos com experiência em atendimento psicoterápico há, em média, 10 anos.

\section{Procedimento de coleta de dados}

A paciente realizou sessão de triagem, em uma clínica privada e foi encaminhada para psicoterapia individual de orientação psicanalítica e avaliação psiquiátrica. A solicitação de participação de Juliana neste estudo foi feita na entrevista de triagem, ocasião em que o Termo de Consentimento Livre e Esclarecido foi lido e assinado. Os registros das sessões foram feitos em vídeo, após concordância da paciente com a filmagem. Os procedimentos de registro em vídeo das sessões foram explicados à paciente que não demonstrou dificuldades em aceitar a gravação. As sessões de psicoterapia ocorreram no consultório da psicoterapeuta em horários agendados conforme acerto entre paciente e terapeuta.

$\mathrm{O}$ atendimento do caso se caracterizou por duas etapas distintas: a primeira delas referiu-se a um período de 12 sessões de psicoterapia, que foram seguidas por um abandono de oito semanas. Após este 
período a paciente procurou a terapeuta e retomaram o processo, que envolveu a realização de mais oito sessões e o abandono definitivo da psicoterapia. Neste estudo, foram avaliadas as 20 sessões que totalizaram o tratamento.

\section{Instrumentos}

Defensive Style Questionnaire (DSQ-40). O DSQ40 é um instrumento desenvolvido por Andrews, Singh e Bond (1993) e seus colaboradores para avaliar os derivativos conscientes dos mecanismos de defesa e traduzido para o Brasil por Blaya et al. (2005). É um questionário objetivo e autoaplicável de 40 questões avaliando 20 defesas de três estilos ou fatores: F1 - imaturo (projeção, agressão passiva, acting-out, isolamento, desvalorização, fantasia-autística, negação, deslocamento, dissociação, cisão, racionalização e somatização); F2 - maduro (sublimação, humor, antecipação e supressão) e F3 - neurótico (anulação, pseudo-altruísmo, idealização e formação reativa). No estudo de Andrade e Shirakawa (2006), a consistência interna do instrumento foi alpha $=.91$.

Symptom Checklist-90-Revised (SCL-90-R). Inventário multidimensional de autoavaliação desenvolvido por Derogatis em 1994 para avaliação de sintomas psicopatológicos, adaptada para o português brasileiro (Laloni, 2001). Trata-se de uma escala do tipo Likert de 5 pontos, composta por 90 itens que visam verificar o padrão psicológico dos respondentes.

Anotações Clínicas. O conteúdo de cada sessão e o processo da psicoterapia foi registrado em forma de relatos sistemáticos de memória da terapeuta. As anotações clínicas, usadas em psicoterapia de orientação psicanalítica, auxiliam na compreensão e estudo do caso.

Adolescent Psychotherapy Q-Set (APQ). O APQ foi desenvolvido no Anna Freud Centre por Calderón (2014) e adaptado para o Brasil por (Benetti et al., 2014, Benetti et al., no prelo) do Psychotherapy Process Q-Set (PQS) de Enrico Jones (2000). Consiste em um instrumento ateórico que tem como medida de avaliação a sessão terapêutica gravada em áudio ou vídeo em toda a sua extensão. Juízes independentes treinados assistem e avaliam a sessão através da distribuição dos itens que incluem a descrição do comportamento do terapeuta $(n=30)$, o comportamento do paciente $(n=40)$ e a interação entre paciente e terapeuta $(n=30)$. Os itens são apresentados em cartões individuais ou na forma de arquivo Excel, compostos pelo item ou afirmação descritiva, seguida de exemplos explicativos. Os cartões são revisados pelos avaliadores e ordenados em uma fileira de nove categorias. Num extremo, ou ao final de uma das pontas da fileira, colocam-se os cartões que o juiz considera serem os mais característicos (categoria 9) da sessão. No outro extremo, ou outra ponta, o avaliador coloca os cartões menos característicos (categoria 1). Demais categorias seguem em um continuum até o centro, onde se colocam cartões neutros (não salientes àquela sessão). As categorias são assim compostas: categorias 9 e 1 ( 5 cartões), categorias 2 e 8 (8 cartões), categorias 7 e 3 (12 cartões), categorias 6 e 4 (16 cartões) e categoria 5 (18 cartões).

\section{Procedimentos de análise dos dados}

A avaliação da paciente foi desenvolvida com base nas sessões psicoterápicas, na avaliação sintomática (SCL-90-R) e defensiva (DSQ-40) realizadas nas sessões iniciais. As anotações clínicas feitas pela terapeuta durante a psicoterapia foram fornecidas na íntegra para a pesquisadora e, posteriormente, associadas às anotações dos juízes avaliadores do APQ sobre as sessões de psicoterapia. Com base neste material foi elaborada a história de vida da paciente e a síntese de cada sessão de psicoterapia.

Para a análise do processo psicoterápico, considerando as variáveis da terapeuta, da paciente e da interação entre paciente e terapeuta, foram utilizadas sessões gravadas em vídeo e avaliadas separadamente conforme o manual do APQ. Participaram desta análise cinco juízes com experiência na avaliação do instrumento. A avaliação de cada juiz foi correlacionada, tendo como referência valores iguais ou acima de 0,7 para se identificar a concordância entre os itens. Quando a correlação entre as avaliações era menor de 0,5 , um terceiro avaliador foi acrescentado. Para as análises subsequentes, utilizou-se a média das pontuações entre os dois avaliadores melhor correlacionados.

A descrição geral do processo de psicoterapia, considerando as variáveis do terapeuta, do paciente e da interação foi realizada através de um ordenamento simples, a partir do cálculo das médias de cada item do APQ, dos itens mais e menos característicos das 20 sessões de atendimento. A análise quantitativa foi construída pelos 10 itens mais e 10 itens menos característicos da psicoterapia, envolvendo Etapa 1 e Etapa 2. No que concerne à análise qualitativa, foram utilizados registros dos juízes avaliadores do processo, associados aos aspectos dinâmicos da paciente, em que posteriormente foi elaborado um entendimento clínico do processo.

\section{Resultados e Discussão}

\section{Análise da paciente}

Juliana buscou tratamento queixando-se de irritabilidade e sintomas depressivos. Conforme apontado 


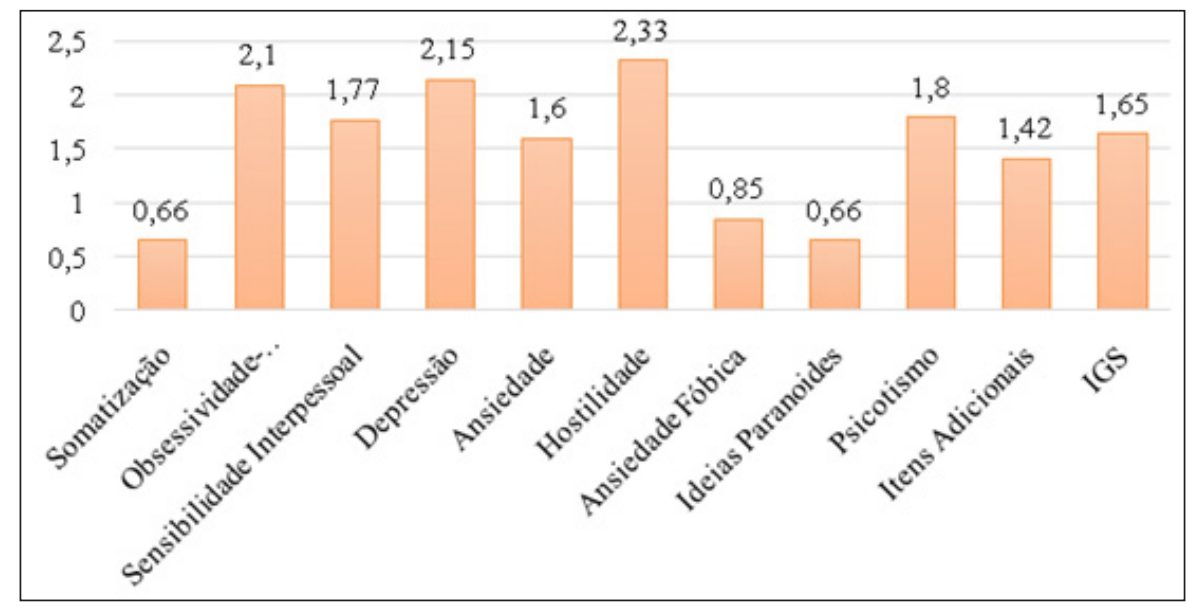

Figura 1. Sintomas predominantes segundo o SCL-90-R.

no SCL-90-R, seus sintomas predominantes abrangiam hostilidade, depressão e obsessividade-compulsividade (Figura 1). Por sua vez, os resultados do DSQ-40 indicam que dentre as manobras defensivas mais utilizadas pelo ego de Juliana evidencia-se uso excessivo de fantasia autística e acting out, prevalecendo um estilo defensivo imaturo e neurótico. Os dados empíricos das medidas de autorrelato associados à história de Juliana sugerem que, ao nível psicodinâmico, as vivências iniciais associam-se a uma dinâmica funcional com déficits significativos. O quadro depressivo originou-se da falta de investimentos libidinais necessários à constituição psíquica.

Por sua vez, a dinâmica da paciente aponta que a negligência materna exigiu de Juliana desenvolver recursos precocemente para suprir esta falta. O ego afetado pela dor defende-se buscando na fantasia a gratificação carente na realidade. Essa manobra defensiva pareceu ter auxiliado e protegido a paciente frente ao reconhecimento de constantes falhas ambientais e relacionais, causadoras de lacunas psíquicas primitivas ao self.

As estratégias despendidas na infância elucidam o funcionamento de Juliana, o trânsito entre fantasia e realidade impedia a jovem de finalizar tarefas circundantes ao amadurecimento egóico. A dificuldade, por exemplo, em adaptar-se às exigências do mercado profissional parecia ser mascarada por uma diminuição de ideais. Nesses momentos o ego esvaziado, se deprimia. Contudo, mesmo enfraquecido, mantinha-se conectado, seu mundo imaginativo não interferia na capacidade que Juliana apresentava de diferenciação da realidade interna e externa, visto que, conseguia distinguir seus conflitos internos de fatos ocorridos na realidade.
O abuso de substâncias no início da adolescência parecia estar mais a serviço de uma necessidade de obtenção da atenção materna. Assim, a dificuldade enfrentada pela paciente referia-se ao amadurecimento egóico, sendo que as defesas imaturas de Juliana obstaculizam a mudança, entende-se que frente a tanta falta, a renúncia necessária ao desenvolvimento emocional para a fase adulta, é adiada pela paciente. Nesse sentido, seu uso esporádico de maconha pareceu ser o combustível do refúgio da realidade do amadurecimento.

\section{Análise do processo}

A primeira etapa da psicoterapia foi constituída por 12 sessões. A Tabela 1 apresenta os itens mais e menos característicos desta etapa. A análise descritiva das sessões através do instrumento APQ identificou os comportamentos do terapeuta, da paciente e da interação entre ambos (Tabela 1). Essa análise aliada às anotações clínicas das sessões permitiu a compreensão do processo de psicoterapia da adolescente.

As sessões iniciais caracterizaram-se pelo engajamento da paciente, que se mostrava disposta e empolgada com a terapia (APQ05r, APQ14r, APQ15r, APQ42r, APQ72) contribuindo ativamente para o processo. Assim, relatou conteúdos significativos referentes às suas ideologias e algumas características pessoais (APQ35) e fatos de sua vida foram associados a conflitos internos (APQ06, APQ09, APQ65, APQ97).

Durante a quarta sessão a terapeuta incentivou (APQ03, APQ31) a paciente a falar sobre um relacionamento afetivo do início de sua adolescência. Ela mostrava-se envergonhada e preocupada com o que a terapeuta pudesse pensar a seu respeito, visto que, este relacionamento se deu com um homem 
TABELA 1

Dez itens mais e 10 itens menos característicos da primeira etapa

\begin{tabular}{|c|c|c|c|}
\hline Item & Descrição & Média & $D P$ \\
\hline 31 & O terapeuta solicita mais informação ou elaboração & 8,50 & 67 \\
\hline 46 & Terapeuta se comunica com o jovem num estilo claro e coerente & 8,25 & ,96 \\
\hline 03 & Observações do terapeuta são destinadas a facilitar fala da paciente & 7,58 & ,90 \\
\hline 09 & Terapeuta trabalha com o jovem para tentar dar sentido à sua experiência & 7,58 & 1,25 \\
\hline 18 & O terapeuta transmite um sentido de aceitação não crítica & 7,25 & 1,65 \\
\hline 06 & Jovem descreve suas experiências emocionais nas interações com pessoas significativas & 7,25 & 1,42 \\
\hline 72 & Jovem se engaja animadamente na discussão de pensamentos e ideias & 6,91 & 1,50 \\
\hline 35 & A autoimagem é um foco de discussão & 6,83 & 1,58 \\
\hline 97 & Terapeuta encoraja reflexão sobre estados e afetos internos & 6,75 & 2,37 \\
\hline 65 & Terapeuta reafirma ou reformula a comunicação do jovem a fim de clarificar seu significado & 6,75 & 1,71 \\
\hline $52 \mathrm{r}^{*}$ & Jovem tem dificuldades com o encerramento das sessões & 2,83 & 1,64 \\
\hline $30 \mathrm{r}$ & Jovem tem dificuldade de iniciar a sessão & 2,75 & 2,22 \\
\hline $14 \mathrm{r}$ & Jovem não se sente entendido pelo terapeuta & 2,75 & 1,60 \\
\hline $20 \mathrm{r}$ & O jovem é provocativo, testa os limites da relação terapêutica & 2,66 & 1,23 \\
\hline $32 \mathrm{r}$ & Jovem adquire uma nova compreensão & 2,41 & 1,92 \\
\hline $51 \mathrm{r}$ & Jovem atribui suas próprias características ao terapeuta & 2,33 & 1,07 \\
\hline $15 \mathrm{r}$ & O jovem não inicia ou elabora os tópicos & 2,33 & 1,82 \\
\hline $05 \mathrm{r}$ & O jovem tem dificuldade em entender os comentários do terapeuta & 2,25 & 1,76 \\
\hline $42 \mathrm{r}$ & Jovem rejeita (versus aceita) os comentários e observações do terapeuta & 2,08 & 1,56 \\
\hline $87 \mathrm{r}$ & Jovem controla a interação com o terapeuta (é controlador) & 1,91 & ,90 \\
\hline
\end{tabular}

* Itens do APQ que se comportaram de maneira reversa, incaracterística.

comprometido e 10 anos mais velho. Esta relação foi definida pela própria paciente como uma desgraça em sua vida e relatada com um aparente distanciamento afetivo do sofrimento envolvido. As intervenções da terapeuta corroboraram para relevantes associações da paciente (APQ09, APQ65, APQ97), visto que a partir do relato desse episódio afetivo causador de tanto sofrimento, ela recordou de um acidente vivenciado na primeira infância em que, aos três anos de idade, cai de um terraço, mas sua ausência demora a ser percebida pela mãe. Fica caída por mais de uma hora, até ser resgatada. Esse acidente trouxe marcas no corpo da paciente que tinha uma cicatriz extensa e aparente em seu braço. Entretanto, utilizava-se do isolamento do afeto, (DSQ-40; escore 3), como um recurso defensivo frente à negligência materna. Essa característica tornava inviável a paciente obter insight durante esta etapa do processo. Assim, a proximidade da integração dos afetos tornou-se tão ameaçadora ao self de Juliana que ela recua, tendo sua primeira falta na psicoterapia. A quinta sessão é caracterizada por uma estruturação mais ativa da terapeuta, que não nomeia a falta, mas busca conhecer a percepção que a paciente tem de seus objetos primários (APQ03, APQ31). A mãe, segundo ela era estranha, esquisita, bipolar, sei lá... Uma mãe que não é presente, mas também não é tão ausente assim, ela tenta dar o melhor mesmo que ela não saiba o que é o melhor, (...) Não sabe o que realmente preciso, quando pequena ela não sabia que precisava me dar atenção (...), mas me deu casa, comida, roupas, nunca me faltou nada. O pai era esquisito e ausente digno de pena pela paciente que o percebia solitário e sem atenção dos filhos. Esta sessão, por sua vez, pareceu trazer a consciência o abandono parental e as possíveis consequências na estruturação de sua personalidade (APQ97, APQ65, APQ87r). Os sentimentos oriundos deste período pareceram assumir uma intensidade que seu aparelho psíquico não dispôs de recursos (APQ32r) para digerir. Então, ocorre mais uma falta.

Inscrições psíquicas da infância pareceram voltar à tona na sexta sessão em forma de descrições do seu mundo imaginativo (APQ30r). Contou sonhos em que acordava assustada, eu sozinha, vendo o mundo acabar e as pessoas nas lojas comprando coisas. Ao mencionar uma amiga imaginária da infância, evidenciou-se que a paciente, utilizava-se de recursos fantasiosos para enfrentamento da realidade. A ansiedade da paciente parece se elevar na sessão sete, a resistência apareceu na vergonha que referiu sentir da terapeuta pelos conteúdos mais primitivos comentados na sessão 
anterior. A terapeuta acolheu a paciente que conseguiu nestes momentos de investimento conectar eventos passados com dificuldades atuais (APQ18, APQ97).

Na sessão 8, mantém-se ainda ansiosa, porém, na medida em que a terapeuta explora conteúdos referentes a experiências emocionais passadas, a paciente consegue associar livremente, fazendo conexões relevantes que são complementadas pela terapeuta (APQ 97) na busca de dar sentido a sua experiência (APQ 09). Na sessão seguinte (sessão 9) mais conteúdos foram explicitados pela paciente (APQ15r, APQ30r, APQ51r), em que envergonhada, a paciente aceitou as intervenções terapêuticas referentes a pensamentos de morte, tristeza e raiva (APQ42r, APQ87r).

A paciente mostrou-se mais silenciosa durante a décima sessão e não fez conexões relevantes com sua conflitiva atual (APQ32r). Assim, não houve evidência de que a paciente tenha conseguido alcançar uma compreensão mais integrada de seu estado depressivo. Comunicou que estaria ausente na próxima sessão em função do seu trabalho, entretanto acaba faltando também à sessão seguinte.

A paciente inicia a décima primeira sessão justificando sua falta, teve uma infecção urinária recorrente. Nesta sessão, a paciente relatou episódios de adoecimentos infantis sem nenhum tipo de continência materna, estes relatos mostraram-se dissociados de afeto e a conduta negligente da mãe foi entendida racionalmente pela paciente. A terapeuta sugeriu que pudessem chamar a mãe para conversar e paciente alegou que talvez ela não conseguisse ir em função do horário de trabalho.

A sessão que antecede o abandono foi caracterizada por breves, mas repetidos momentos de silêncio. A paciente apresentava-se esvaziada, rígida e resistente; a terapeuta, por sua vez, direcionou intervenções que visavam explorar vivências infantis e estados emocionais (APQ 97). Entretanto, a paciente resistiu e não encontrou recursos internos capazes de tolerar tamanha atenção disponibilizada pela terapeuta (APQ18).

A paciente ausentou-se do processo por dois meses. Entretanto, durante o período em que esteve afastada da psicoterapia, Juliana enviava esporádicas mensagens telefônicas expressando desejo de retornar ao tratamento psicoterápico, até que foi efetivado contato telefônico com a terapeuta e retomaram as sessões. A sessão de retomada ao processo foi caracterizada pela discussão da interrupção, iniciando a segunda etapa da psicoterapia que foi composta por 8 sessões (Tabela 2).

Juliana verbalizou que a terapia estava fazendo com que ela refletisse acerca de fatos tristes de sua vida, e que considerava desnecessário, visto que, já compreendeu tudo e que o que passou, passou (APQ53r, APQ78r, APQ37). A terapeuta assumiu uma postura mais ativa (APQ17), pontuou que a paciente desconfiava da

TABELA 2

Dez itens mais e 10 itens menos característicos da segunda etapa

\begin{tabular}{|c|c|c|c|}
\hline Item & Descrição & Média & $D P$ \\
\hline 31 & O terapeuta solicita mais informação ou elaboração & 8,37 & ,70 \\
\hline 37 & Terapeuta permanece empático quando confrontado com intensos sentimentos ou impulsos do jovem & 7,87 & ,97 \\
\hline 17 & Terapeuta estrutura ativamente a sessão & 7,75 & 1,06 \\
\hline 09 & Terapeuta trabalha com o jovem para tentar dar sentido à sua experiência & 7,50 & 1,03 \\
\hline 97 & Terapeuta encoraja reflexão sobre estados e afetos internos & 7,50 & ,88 \\
\hline 46 & Terapeuta se comunica com o jovem num estilo claro e coerente & 7,37 & 1,57 \\
\hline 35 & A autoimagem é um foco de discussão & 7,37 & 1,12 \\
\hline 03 & Observações do terapeuta são destinadas a facilitar a fala do jovem & 7,37 & ,95 \\
\hline 24 & Jovem demonstra capacidade de vincular estados mentais a ações ou comportamentos & 7,25 & 1,37 \\
\hline 38 & O terapeuta e o jovem demonstram uma compreensão mútua em relação a eventos ou sentimentos & 7,12 & 1,15 \\
\hline $53 \mathrm{r}$ & Jovem discute experiências como se estivesse distante de seus sentimentos & 2,87 & 1,34 \\
\hline $93 \mathrm{r}$ & Terapeuta se abstém de tomar posição em relação aos pensamentos ou comportamentos do jovem & 2,62 & 1,92 \\
\hline $78 \mathrm{r}$ & Jovem procura aprovação, afeição ou simpatia do terapeuta & 2,62 & 1,35 \\
\hline $67 \mathrm{r}$ & Jovem demonstra dificuldade para se concentrar ou manter a atenção durante a sessão & 2,37 & 1,71 \\
\hline $51 \mathrm{r}$ & Jovem atribui suas próprias características ao terapeuta & 2,37 & 2,32 \\
\hline $15 \mathrm{r}$ & O jovem não inicia ou elabora os tópicos & 2,25 & 1,69 \\
\hline $14 \mathrm{r}$ & Jovem não se sente entendido pelo terapeuta & 2,25 & 1,75 \\
\hline $87 \mathrm{r}$ & Jovem controla a interação com o terapeuta (é controlador) & 1,87 & 1,28 \\
\hline $52 \mathrm{r}$ & Jovem tem dificuldades com o encerramento das sessões & 1,87 & 2,25 \\
\hline $05 \mathrm{r}$ & O jovem tem dificuldade em entender os comentários do terapeuta & 1,37 & ,92 \\
\hline
\end{tabular}


atenção recebida, associando também a interrupção da psicoterapia com a parada da medicação, acredito que a medicação ajudaria a te deixar mais forte e assim poderíamos ficar mais próximas. A terapeuta mostrou-se compreensiva quanto à dificuldade de aderência a psicoterapia, sei o quanto este processo é dolorido para ti, talvez por isso, tenha que ir e vir (APQ38, APQ09, APQ37).

Conteúdos bastante significativos foram explorados nas sessões 14 e 15 (APQ24, APQ35) acerca da falta de investimento libidinal materno e de que como, desde cedo, aprendeu a se cuidar (APQ24, APQ51r). Frente ao acesso aos conflitos primitivos, a terapeuta convidou a paciente a pensar sobre a raiva que podia ter da mãe e do pai e a integração de afetos mais agressivos tornou-se o foco da sessão (APQ97, APQ17, APQ09, APQ53r).

$\mathrm{O}$ temor novamente ressurgiu e Juliana atuou novamente ausentando-se da sessão seguinte. $\mathrm{Na}$ sessão 16 referiu estar fumando maconha todos os dias juntamente com o irmão. A droga parecia anestesiar a dor do abandono quando fumo tudo fica melhor, a vida mais leve e mais engraçada, eu e o mano conversamos muito quando fumamos, acho até que tenho vários insights. A função terapêutica passou a ser da maconha e Juliana faltou novamente à psicoterapia. A sessão 17 foi caracterizada pela exploração das fantasias da paciente. A partir de intervenções da terapeuta (APQ97, APQ09) a paciente associou livremente e relatou com riqueza de detalhes o mundo imaginário que a acompanhou durante a infância e adolescência (APQ67r, APQ15r).

Frente às interpretações da terapeuta quanto ao seu temor em acessar conteúdos tristes Juliana justificou: sempre quando estou muito mal, penso que existem livros e seriados para assistir, a continência nunca encontrada nos objetos primários ficou a cargo da ficção (APQ97, APQ09). Contudo, frente à discussão sobre o episódio do acidente vivenciado aos 3 anos de idade, ela recompõe a defesa: qual o problema com o tombo, eu só quebrei o braço (APQ03, APQ 09, APQ17, APQ46, APQ05r).

O silêncio invadiu as últimas sessões da segunda etapa do processo. Os pensamentos sobre morte voltaram à tona e anunciaram o abandono que foi explorado pela terapeuta. Frente a esta observação, a paciente associou e discutiu sobre as duas "Julianas" existentes, uma do mal e outra do bem, o pior é que quando uma aparece a outra demora a perceber, e tem uma que não gosta de falar em coisas tristes (APQ37, APQ38, APQ93r, APQ67r, APQ14r, APQ05r).

A sessão 19 foi focada na não tolerância em vivenciar angústias que geraram atuações que elucidaram o abandono. Seus pensamentos de morte prevaleceram e de fato pareceu que a Juliana que não gostava de falar em coisas tristes predominou e ambas "Julianas" fugiram da psicoterapia, contrariando o pedido da terapeuta: Fico preocupada com a possibilidade de ir embora, o problema é que se tu for, as Julianas vão contigo, seria bom se tolerasse a psicoterapia e pudesse deixar essa Juliana mal aqui comigo (APQ37, APQ93r).

A sessão 20, precedente ao abandono da psicoterapia, foi caracterizada por pouca exploração de temáticas relacionadas a seus conflitos, a paciente mostrou-se deprimida, paralisada e sem perspectivas. A pouca capacidade egóica na obtenção de recursos propulsores ao amadurecimento buscou um rearranjo atuado, melhor dizendo, tatuado no corpo: Juliana mostrou à terapeuta a frase tatuada no braço que havia feito naquela semana, um brinde para nunca crescermos. Esta pareceu ser o anúncio do abandono.

Em síntese, com base na análise da descrição da psicoterapia e no entendimento clínico do processo pode-se evidenciar que Juliana abandona a psicoterapia, acompanhada por sua sintomatologia e amparada por defesas imaturas. Ao longo do processo, observa-se uma paciente ativa, que narra conteúdos relativos a seus conflitos, disposta a quebrar silêncios e expressando sentir-se entendida pela terapeuta. Esta, por sua vez, é clara em suas intervenções e predominantemente encoraja a exploração de afetos e estados internos ligados às experiências iniciais com objetos primários. Todavia, o contato com esses afetos ligou-se a um aumento de ansiedade que não foi tolerado por Juliana.

Essa incapacidade de tolerar uma mudança em sua percepção acerca da origem de sua depressão, ou seja, de insight acerca de suas vivências, tornam o trabalho na sessão difícil - afinal foi apenas um tombo, ao referir-se a um acidente grave resultante de falha de supervisão parental. Dessa forma, analisando-se a psicoterapia pelo viés de um processo, observa-se que a paciente apresenta falhas na conexão dos afetos com seus conflitos internos. Ainda que de forma incipiente, ela buscou conectar seu sofrimento com a falta de investimento libidinal dos objetos primários. Entretanto, a falta do registro de uma relação de cuidados e a impossibilidade de obtenção de insight pode ter contribuído para o afastamento de Juliana da psicoterapia.

Em um estudo inglês realizado com 55 adolescentes entre 11 e 19 anos, a capacidade de insight foi identificada como influenciando tanto a aderência terapêutica como o desfecho clínico (Gatta et al., 2010). Da mesma forma, um estudo nacional com pacientes que abandonaram a psicoterapia psicanalítica na 28aㅗ sessão aponta que dentre outras causas subjacentes ao abandono está a baixa capacidade de insight (Jung, Serralta, Nunes, \& Eizirik, 2015). Ao explorar intervenções promotoras de insight no processo terapêutico, McAleavey e 
Castonguay (2013) referem que muitas vezes a persistência do terapeuta em realizar intervenções interpretativas sem ajustar-se à reação do paciente pode associar-se a resultados negativos, tais como o abandono.

No presente caso, conforme a psicoterapia foi exigindo de Juliana uma posição de reflexão sobre a conflitiva interna, as defesas frente à ansiedade provocada pelas vivências de abandono acionaram mecanismos de negação e acting out. Esses foram expressos ao minimizar os conflitos e ao incorrer em faltas. Ainda que a compreensão da paciente permita a identificação desse funcionamento, resta a questão do lugar do terapeuta no processo. Nessas situações, levar em conta a capacidade de insight do paciente em relação à tolerância de vivências afetivas conflitivas é uma estratégia importante frente às ameaças de rupturas, posição que o terapeuta deve assumir para manter o vínculo de trabalho. Assim, observa-se que o trabalho interpretativo, face às resistências, pode não ter alcançado o objetivo de aliviar a angústia.

\section{Considerações finais}

Este artigo evidenciou a consonância de dados empíricos obtidos através da análise do processo psicoterápico com características dinâmicas e clínicas da paciente investigada, apontando para a viabilidade da clínica se integrar a pesquisa. A psicoterapia convidou
Juliana a reatualizar na transferência sua conflitiva de base. Entretanto, a internalização de uma figura materna negligente vai de encontro a uma terapeuta disponível, continente e que se esforçou em promover novos significados aos conflitos. A vivência dessa experiência mais acolhedora não foi tolerada por Juliana.

Assim, a não aquisição de insight da paciente durante as sessões, aliada a um estilo defensivo imaturo constituíram a base do abandono psicoterápico. Nesse sentido, evidencia-se a importância do terapeuta estar atento à capacidade interna de elaboração dos conflitos pelo adolescente ao longo da psicoterapia para que possa prevenir rupturas da aliança terapêutica através da utilização de intervenções específicas ao caso. Contudo, destaca-se a limitação da presente pesquisa, tal como o fato de ter se baseado em um estudo de caso e frente aos diversos vértices de compreensão do processo psicoterápico, que possibilitariam análises complementares do fenômeno.

Ainda assim, a viabilidade de contemplar a avaliação de um processo tão subjetivo com medidas empíricas amplia as possibilidades de entendimentos das psicoterapias. Posturas investigativas adotadas pelos clínicos e pesquisadores são, portanto, medidas que podem contribuir para a melhoria dos atendimentos psicoterápicos, destacando-se a importância da realização de pesquisas que visem à análise em profundidade dos processos psicoterápicos.

\section{Referências}

Adesse, D. B., Santos, V. L. A., \& Cardoso, M. R.(2014). Drogadicção e adolescência: o "corpo do transbordamento". Revista Latinoamericana de Psicopatologia Fundamental, 17(3), 544-556. https://doi.org/10.1590/14154714.2014v17n3p544-11

American Psychiatric Association (2002). DSM-IV-TR, Manual Diagnóstico e Estatístico de Transtornos Mentais (4 ${ }^{\text {th }}$ ed., rev.). Porto Alegre: ArtMed.

Andrade, M. \& Shirakawa, I. (2006). Versão brasileira do Defense Style Questionnaire (DSQ) de Michael Bond: problemas e soluções. Revista de Psiquiatria do Rio Grande do Sul, 28(2), 144-160. https://doi.org/10.1590/S010181082006000200007

Andrews, G., Singh, M., \& Bond, M. (1993). The Defense Style Questionnaire. The Journal of Nervous and Mental Disease, 181, 246-256. Retrieved from: jonmd/Abstract/ 1993/04000/The_Defense_Style_Questionnaire.6.aspx. https://doi.org/10.1097/00005053-199304000-00006

Benetti, S. P. C., Esswein, G. C., Bernardi, G., Midgley, N., Calderon, A. (2014). Adolescent Psychotherapy Q-Set (APQ): Prototypes of psychoanalytic psychotherapy and cognitive behavioral therapy. Book of Abstracts Society for Psychotherapy Research $45^{\text {th }}$ International Annual Meeting. Copenhagen, Denmark.

Benetti, S. P. C., Esswein, G. C., N., Bohn da Silva, Bernardi, G., \& Calderon, A. (no prelo). Pesquisa de processo em psicoterapia na adolescência: Adaptação do instrumento APQ. PsicoUSF (Impresso).

Blaya, C. (2005). Tradução, adaptação e validação do Defensive Style Questionnaire (DSQ-40) para o português brasileiro. [Dissertação de Mestrado]. Faculdade de Medicina, Universidade Federal do Rio Grande do Sul. Porto Alegre, Brasil. Retrieved from: http://www.lume.ufrgs.br/bitstream/handle/10183/5601/000472900.pdf?sequence=1

Calderón, A. (2014). Development and validation of the Adolescent Psychotherapy Q-set (APQ). [Doctoral thesis]. University College of London, London, UK. Retrieved from: http://discovery.ucl.ac.uk/1453282/

Derogatis, L. R. (1994). Symptom Checklist-90-R (SCL-90-R): administration, scoring, and procedures manual ( $\left.3^{\mathrm{a}} \mathrm{ed}.\right)$. Minneapolis: National Computer Systems, USA.

Edlund, J. N. \& Carlberg, G. (2016). Psychodynamic psychotherapy with adolescents and young adults: Outcome in routine practice. Clinical Child Psychology and Psychiatry, 21, 66-80. https://doi.org/10.1177/1359104514554311 
Gastaud, M. B., Basso, F., Soares, J. P. G., Eizirik, C. L., \& Nunes, M. L. T. (2011). Preditores de não aderência ao tratamento na psicoterapia psicanalítica de crianças. Revista de Psiquiatria do Rio Grande do Sul, 33(2), 109-115. Retrieved from: <http://www.scielo.br/pdf/rprs/v33n2/1328.pdf>. https://doi.org/10.1590/S0101-81082011005000011

Gatta, M., Spoto, A., Testa, P., Svanellini, L., Lai, J., Salis, M., Sauma, M., \& Battistella, P. (2010). Adolescent insight within the working alliance: a bridge between diagnostic and psychotherapeutic processes. Adolescent Health, Medicine and Therapeutics, 1, 45-52. https://doi.org/10.2147/AHMT.S9323

Gerber, A., Kocsis, J., Milrod, B., Roose, S., Barber, J., Thase, M., ..., \& Leon, A. (2011). A quality-based review of randomized controlled trials of psychodynamic psychotherapy. American Journal of Psychiatry, 168, 19-28. https:// doi.org/10.1176/appi.ajp.2010.08060843

Haan, A. M., Boon, A. E., Jong, J. T. V. M., Hoeve, M., \& Vermeiren, R. J. M. (2013). A meta-analytic review on treatment dropout in child and adolescent outpatient mental health care. Clinical Psychology Review, 33, 698-711. https://doi. org/10.1016/j.cpr.2013.04.005

Jones, E. E. (2000). Therapeutic action: a guide to psychoanalytic therapy. Northvale, NJ: Jason Aronson.

Jung, S. I., Serralta, F. B., Nunes, M. L. T., \& Eizirik, C. L. (2015). Desistência e conclusão em psicoterapia psicanalítica, um estudo qualitativo de pacientes de Porto Alegre, Brasil. Revista Brasileira de Psicoterapia, 17(1), 25-40.

Laloni, D. T. Escala de avaliação de sintomas-90-R SCL-90-R: adaptação, precisão e validade. 2001. [Tese de Doutorado]. Pontifícia Universidade de Campinas, Campinas, 2001.

Macedo, Mônica Medeiros Kother, Baldo, Mariana Aguilar, Santos, Rafael Lisboa dos, Ribas, Renata Freitas, Silva, Sander Machado da, \& Gonçalves, Thomás Gomes. (2011). Motivos de busca de atendimento psicológico por adolescentes em uma clínicaescola. Psicologia: teoria e prática, 13(2), 63-75. Recuperado em: 09 jan. 2017, de http:// pepsic.bvsalud.org/scielo.php?script=sci_arttext\&pid=S1516-36872011000200005\&lng=pt\&tlng=pt

McAleavey, A. A. \& Castonguay, L. G. (2013). Insight as a common and specific impact of psychotherapy: therapistreported exploratory, directive, and common factor interventions. American Psychological Association, 1-12. https:// doi.org/10.1037/a0032410

McLeod, J. \& Elliott, R. (2011). Systematic case study research: A practice and oriented introduction to building an evidence base for counselling and psychotherapy. Counselling and Psychotherapy Research, 11, 1-10. https://doi.or $\mathrm{g} / 10.1080 / 14733145.2011 .548954$

Merikangas, K. R., He, J., Burstein, M., Swanson, S. A., Avenevoli, S., Cui, L.,.., \& Swendsen, J. (2010). Lifetime Prevalence of Mental Disorders in US Adolescents: Results from the National Comorbidity Study-Adolescent Supplement. Journal of the American Academy of Child \& Adolescent Psychiatry, 49(10), 980-989. https://doi. org/10.1016/j.jaac.2010.05.017

Organização Mundial da Saúde. (2015). Adolescent health research priorities: report of a technical consultation. 22p.

Palmer, R., Nascimento, L. N., \& Fonagy, P. (2013). The state of the evidence base for psychodynamic psychotherapy for children and adolescents. Child and Adolescent Psychiatric Clinics of North America, 22, 149-214. https://doi. org/10.1016/j.chc.2012.12.001

Serralta, F. B., Nunes, M. L. T., \& Eizirik, C. L. (2011). Considerações metodológicas sobre o estudo de caso na pesquisa em psicoterapia. Estudos de Psicologia, 28(4), 501-510. https://doi.org/10.1590/S0103-166X2011000400010

Shedler, J. (2012). The efficacy of psychodynamic psychotherapy. In R. A. Levy, J. S. Ablon, \& H. Kächele (Eds.). Psychodynamic Psychotherapy Research (pp. 9-25). New York, NY: Humana Press. https://doi.org/10.1007/978-160761-792-1 2

Ormhaug, S. M. \& Jensen, T. K. (2016): Investigating treatment characteristics and first-session relationship variables as predictors of dropout in the treatment of traumatized youth. Psychotherapy Research, 27, 1-15. https://doi.org/ 10.1080/10503307.2016.1189617

Stewart, P. K., Steele, M. M., \& Roberts, M. C. (2012). What happens in therapy? Adolescents' expectations and perceptions of psychotherapy. Journal of Child and Family Studies, 23(1), 1-9. https://doi.org/10.1007/s10826-012-9680-3

Valverde, B. S. C. L., Vitalle, M. S. S., Sampaio, I. P. C., \& Schoen, T. H. (2012). Levantamento de Problemas Comportamentais/emocionais em um ambulatório para adolescentes. Paidéia, 22(53), 315-323.

Weitkamp, K., Daniels, J. K., Hofmann, H., Timmermann, H., Romer, G., \& Wiegand-Grefe, S. (2014). Psychoanalytic psychotherapy for children and adolescents with severe depressive psychopathology: Preliminary results of an effectiveness trial. Psychotherapy, 51, 138-147. https://doi.org/10.1037/a0034178

\section{Autores:}

Silvia Pereira da Cruz Benetti - Doutora, Universidade do Vale do Rio dos Sinos.

Maria Cristina Vieweger de Mattos - Mestre, Universidade do Vale do Rio dos Sinos.

Nathália Bohn da Silva - Universidade do Vale do Rio dos Sinos.

Aline Alvares Bittencourt - Mestre, Universidade do Vale do Rio dos Sinos.

Endereço para correspondência:

Silvia Pereira da Cruz Benetti

Rua Riveira, 150/301

90670-160, Porto Alegre, RS, Brasil

<sbenetti@unisinos.br>

Recebido em: 03.08.2016

Aceito em: 19.01.2017 